\title{
A moving model rig with a scale ratio of $1 / 8$ for high speed train aerodynamics
}

\author{
Qian-Suo Yang*, Jun-Hao Song, Guo-Wei Yang \\ Key laboratory for Mechanics in Fluid Solid Coupling Systems, Institute of Mechanics, Chinese Academy of Sciences, Beijing 100190, China
}

\section{A R T I C L E I N F O}

\section{Article history:}

Received 8 July 2015

Received in revised form

2 March 2016

Accepted 6 March 2016

Available online 16 March 2016

Keywords:

Moving model rig

Acceleration

Deceleration

Magnetic brake

Air gun

Compressed air

\begin{abstract}
A B S T R A C T
To achieve an accurate aerodynamic optimization of the outline of a high speed train and an experimental simulation of the meeting of two trains, especially in a tunnel, a train model in a moving model rig (MMR) should feature a large scale ratio and be capable of accelerating to the real Mach number. For this purpose, an MMR is developed for the acceleration, testing, and deceleration of a train model along opposite directions. To meet the railway standards of China, the distance between the centers of the two tracks is set to $625 \mathrm{~mm}$ for the scale ratio of $1 / 8$. The powers of the train models originate from compressed air and the brake force from the motion of the permanent magnets relative to the motionless steel plates along the tracks. The compressed air from an air gun with an air chamber pushes a set of pistons in an accelerating tube forward. These pistons tow a trailer through a towrope, and this trailer drives the train model up to a certain speed. The initial pressure of the compressed air determines the speed of the train model, and the brake distance depends on the kinetic energy of the model and the weight of magnets on the bottom of the model. The corresponding experimental measurements are presented in this work. Two train models weighing 265 and $106 \mathrm{~kg}$ can be accelerated to speeds of 401 and $507 \mathrm{~km} / \mathrm{h}$, respectively, within a brake distance of $70 \mathrm{~m}$. Thus, the train model with one or several cars can have a scale ratio of at least $1 / 8$. Additional experimental results on the evolution of the pressure wave on the tunnel wall are introduced to demonstrate the repeatability of the experiment on the train model passing through the tunnel model.
\end{abstract}

(c) 2016 Elsevier Ltd. All rights reserved.

\section{Introduction}

As trains accelerate, aerodynamic resistance and noise strength all increase. When train speed is over $300 \mathrm{~km} / \mathrm{h}$, about $80 \%$ of the train's power is used to overcome aerodynamic resistance (Schetz, 2001; Raghunathan et al., 2002). Therefore, the aerodynamic shape of high speed trains should be optimized to reduce air resistance and noise. In other instances, aerodynamic processes such as the passing of trains through tunnels, bridges, and buildings lead to sudden changes in the pressure on train surfaces. Moreover, when two trains meet in an open air environment or in a tunnel, the intricate fluctuation of pressures on the trains disturbs the smooth motion of the trains to a certain extent because of the interaction between the train aerodynamics and the propagation of the pressure wave in the tunnel (Schetz, 2001; Raghunathan et al., 2002; Tian, 2007; Wagner et al., 2014). Therefore, these aerodynamic processes deserve theoretical and

\footnotetext{
* Correspondence to: Institute of Mechanics, Chinese Academy of Sciences, No.15 Bei Si Huan Xi Lu, Hai Dian District, Beijing, 100190, China. Tel.: +86 01082544006. E-mail address: qsyang@imech.ac.cn (Q.-S. Yang).
}

experimental investigation to ensure the safety and stability of high speed trains.

A moving model rig (MMR) is usually more advantageous in experimental studies on train motion than in wind tunnel experiments. First, given the relative motion of trains on road surfaces, MMRs are highly suitable for experiments simulating actual scenes. Second, wind tunnel experiments for trains can only simulate processes involving the movement of a single train in a simple environment, such as in open air. Experimental simulations of trains passing through tunnels and buildings and over bridges can be conducted only with MMRs. Finally, meetings of trains can only be simulated with MMRs for experimental research. Therefore, this type of technology has gradually become an important experimental measurement device for the research of high speed trains. Moreover, the development and construction of MMR devices with large scale ratios and high simulation speeds are excellent bases of accurate experimental simulations, especially those on the dynamic characteristics of high speed trains.

Several MMRs for studying the dynamics of high speed trains have been constructed all over the world. In the UK, the TRAIN rig featuring a test section of $50 \mathrm{~m}$ can accelerate a train model with a scale ratio of $1 / 25$ to a maximum speed of $270 \mathrm{~km} / \mathrm{h}$, with the train 
model driven by two sets of rubber catapults for two rigs (Johnson and Dalley, 2002). The German Aerospace Center employs the simulation equipment called the TSG, in which a train model is driven by a hydro-pneumatic device and passes through a tunnel or wind area at a constant speed. The speed of the model can be adjusted up to the maximum of $360 \mathrm{~km} / \mathrm{h}$. The length of its track is $60 \mathrm{~m}$, and the equipment can be outfitted with a tunnel simulation device. The circulating wind tunnel can provide winds of up to $90 \mathrm{~km} / \mathrm{h}$ in the direction perpendicular to the track (http://scart. dlr.de/site/test-facilities/tsg/). The scale ratio of the model with a maximum weight of $10 \mathrm{~kg}$ ranges from $1 / 100$ to $1 / 20$. At the end of the track, soft plastic balls on the ground are used to decelerate the model. With crosswind at different speeds, this experimental equipment is mainly utilized to measure the pressure wave at the inlet and outlet of the tunnel as the train model passes through with different outlines. The MMR of the Key Laboratory of Track Traffic Safety, Central South University measures $164 \mathrm{~m}$ in length. Two models can move along opposite directions for the simulation of two trains crossing each other. The scale ratio ranges from $1 / 20$ to $1 / 16$. The train model accelerates through two levels of a pulley mechanism up to a maximum speed of $500 \mathrm{~km} / \mathrm{h}$. The brake system of the device adopts a combination of several types of mechanical brake systems, including friction brake, piston brake, and brake disc, to ensure the smooth deceleration of the train model (Zhou et al., 2014). A number of devices based on the air gun principle have been built to study the characteristics of tunnel pressure wave for high speed trains (Ozawa and Maeda, 1988; Takayama et al., 1995; Sasoh et al., 1998; Demmenie et al., 1998; Doi et al., 2010; Endo et al., 2014). However, for an accurate aerodynamic simulation in the design and optimization of trains with complex outlines in the field of engineering, train models should have scale ratios larger than 1/10 and reach the same speed as that of actual trains (Peters, 1983; Schetz, 2001; Tian, 2007).

The size of a train model is known to be directly proportional to its mass and kinetic energy. Thus, powerful drive capabilities and effective brakes are requisite for MMRs with large-scale train models. The advantages, sophistication, and repeatability of MMRs are obviously determined by the principles of acceleration and deceleration of train models, as well as by the scale ratio and speed of such train models. Moreover, the aforementioned two processes depend on the driving and braking mechanisms of train models. Yang et al. (2013) successfully developed technologies for the acceleration and deceleration of small train models, whose driving power and braking force originate from the release of compressed air and the motion of magnets on the model relative to the steel plates on the ground, respectively.

In the present work, we optimize the above principles in our development of an MMR with a scale ratio of $1 / 8$. Specifically, this work reports the principles and corresponding parameters of the proposed MMR. In this MMR, the length of the test section is $60 \mathrm{~m}$, and the train models weighing 265 and $106 \mathrm{~kg}$ are not only accelerated to the maximum speeds of 401 and $507 \mathrm{~km} / \mathrm{h}$ but also decelerated smoothly by the magnetic force within $70 \mathrm{~m}$. Some experimental simulations on the passing of a train through a tunnel are reported to reveal the good repeatability of the experiments under the same initial compressed air pressure in the MMR.

\section{Experimental device}

The proposed MMR is established in Huai Rou District, Beijing. In the MMR, two train models along different tracks in reverse directions intersect to demonstrate the meeting of two trains, a photograph for this MMR is given in Fig. 1. The corresponding structures and working principles are shown in Fig. 2(a) and (b). To

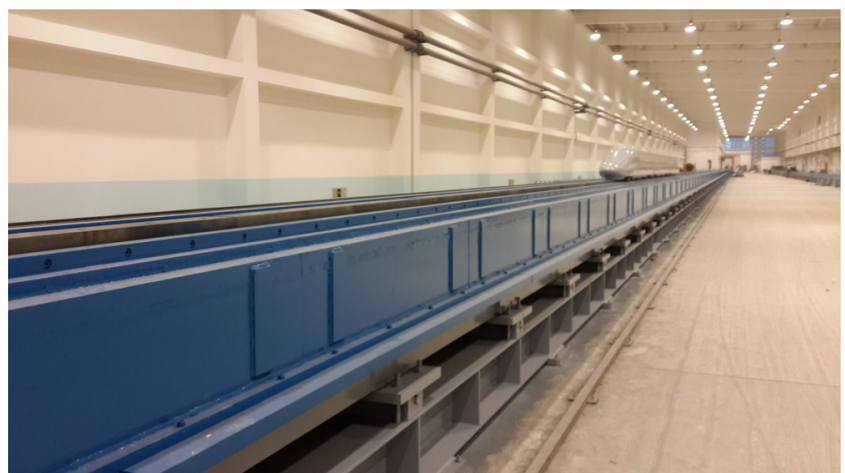

Fig. 1. Photograph of the MMR with a scale ratio of $1 / 8$ in Huai Ruo District, Beijing.

simulate these cases with a scale ratio of $1 / 8$, the distance of the track center is set to $625 \mathrm{~mm}$ to represent the actual distance of a $5 \mathrm{~m}$ railway track in China. In this device, the length of the upper track for the train model is $264 \mathrm{~m}$, and the distance between two tracks is $140 \mathrm{~mm}$; for the lower track, the values for the trailer are $110 \mathrm{~m}$ and $100 \mathrm{~mm}$, respectively. The train model and trailer are respectively restricted to the upper and lower tracks, but they are set to move freely along the tracks with the slide blocks of the model and the trailer, respectively. The vertical distance between the two tracks is $75 \mathrm{~mm}$. The schematic diagram for the acceleration and deceleration of the train model is shown in Fig. 2(b). The acceleration length is $58.5 \mathrm{~m}$, and the deceleration distance is $51 \mathrm{~m}$ for the trailer. The lengths of the acceleration and deceleration tubes for the pistons are $50 \mathrm{~m}$ and $48 \mathrm{~m}$, respectively, and they are with the same diameter of $204 \mathrm{~mm}$. An exit tube with a length of $6 \mathrm{~m}$ and a diameter of $204 \mathrm{~mm}$ is provided between the two tubes above, and 24 rectangular holes with a dimension of $160 \times 200 \mathrm{~mm}^{2}$ downward are averagely distributed for the exit of compressed air.

Initially, the end of the acceleration tube for the input of compressed air is sealed with a flange, but a small hole with a diameter of $20 \mathrm{~mm}$ is provided at the center of the flange for the towrope. The towrope is connected to a piston with a diameter of $203 \mathrm{~mm}$ in the acceleration tube and the trailer on the lower track, as shown in Fig. 2(a) and (b). Another piston is located between the stationary flange and the moving piston. The second piston can freely move along the towrope in the tubes because the towrope passes through the hole at the center of this piston. In this device, the length of the towrope is equal to that of the lower track and is shorter than the sum of the lengths of the acceleration tube, exit tube, and deceleration tube. This setting ensures that the first piston stops in the deceleration tube as the trailer reaches the end of the lower track.

The compressed air for driving the pistons comes from an air gun with a gas chamber of $3.0 \mathrm{~m}^{3}$ and its schematic diagram is shown in Fig. 3(a). The cross section of the piston in the stainless steel cylinder is four times that of the plug at the exit of the gas chamber. To operate this air gun safely, three solenoid valves are used in controlling the entry of the compressed air into the chamber, the connection and isolation of the regions on the two sides of the piston in the cylinder, and the sudden release of the compressed air stored in the cylinder.

The process for the storage of the compressed air is as follows. The No. 2 solenoid valve is first opened to connect the regions on the two sides of the piston and thereby cause the plug to shut down the air chamber as the compressed air from the No. 1 solenoid valve enters the cylinder and drives the piston. As a result of the pressure difference of both sides of the plug, the plug is sealed for the chamber, and the compressed air is stored and increased as needed to achieve the desired speed of the train model. After the 
a

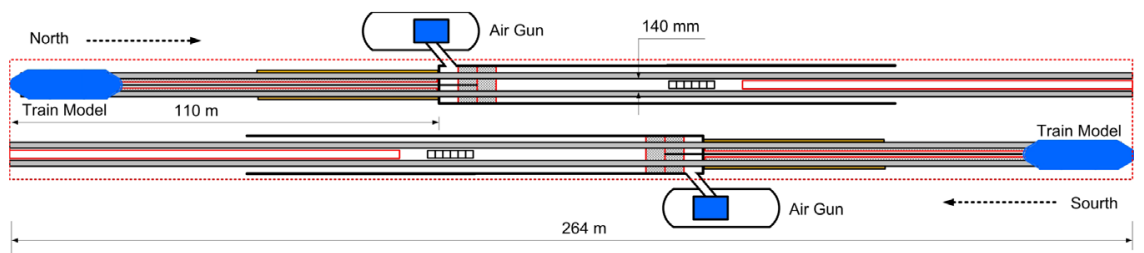

b

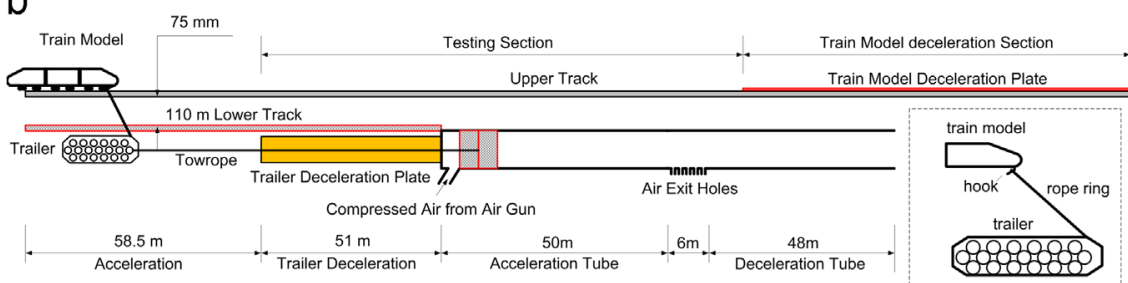

Fig. 2. Schematic diagram and some structural parameters of the MMR with two train models along opposite directions: (a) vertical view and (b) lateral view.
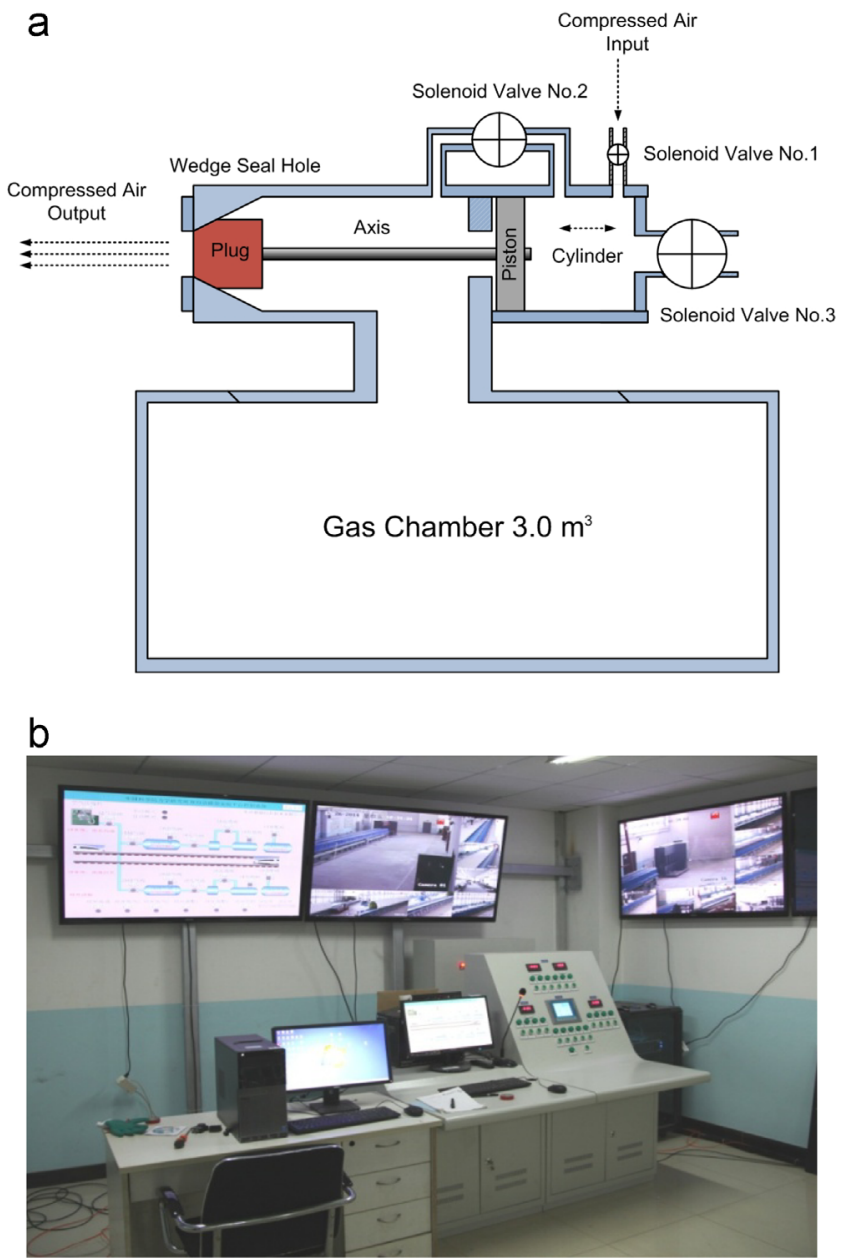

Fig. 3. (a) Schematic diagram of an air gun with a compressed air chamber of $3.0 \mathrm{~m}^{3}$ and (b) photograph of the operation system of the MMR.

storage of the compressed air, the No. 2 solenoid valve should be shut down. The release of the gas is controlled by the No. 3 solenoid valve. As this valve opens up, the pressure in the cylinder suddenly decreases because of the exit of the compressed air. A great drag is produced because of the pressure difference of both sides of the piston. The plug is rapidly opened as this force is larger than the force from the pressure difference of both sides of the plug. Thus, the compressed air in the gas chamber is released and enters the acceleration tube. In the operation for the meeting of the two train models at the test section along the opposite directions, the delay time between the electric pulses for the opening of the No. 3 solenoid valves of two air guns is adjusted by the operation system of this MMR, as shown in Fig. 3(b).

As the air from the air gun suddenly enters the acceleration tube between the pistons and the flange, the pressure on the pistons from the compressed air pushes them to accelerate forward. The leaking from the edge of the pistons and the small hole at the center of the flange are neglected. In this device, the area of the gap between the pistons and acceleration tube and the space of the small hole at the center of the flange and towrope is about $8 \mathrm{~cm}^{2}$, with the diameter of the towrope being $14 \mathrm{~mm}$. This area is smaller than the cross section of the tube with an inner diameter of $200 \mathrm{~mm}$ between the air gun and the acceleration tube as well as that between the acceleration tube and the pistons.

The power from the pistons is transmitted to the trailer with the towrope, and the trailer drives the train model forward. This acceleration process gradually ends as the pistons enter the exit tube because the pressure on the pistons gradually weakens as the compressed air spills through the rectangular holes on the exit tube. At this point, the trailer enters the trailer deceleration section and begins to decelerate. However, the train model maintains a uniform motion because of its inertia. Thus, the speed of the model differs from the trailer and results in their automatic separation, in which a strong hook and a soft rope ring are fixed on the bottom of the train model and on top of the trailer, respectively. With the hook and rope ring, the trailer draws the train model as they speed up in the acceleration section. As the trailer slows down behind the train model, the rope ring falls over and separates from the hook, as shown in the inset of Fig. 2(b).

In the trailer deceleration section, the motion of the permanent magnets on the trailer relative to the deceleration steel plate on the ground induces a brake force (Yang et al., 2013; Jirout et al., 2009; Jou et al., 2006; Maclatchy et al., 1993; Ma et al., 2010). Thus, the trailer slows down and finally stops, as illustrated in Fig. 4. The pistons and towrope are also decelerated to stop because of the connection of the trailer to the pistons though the towrope.

To simulate the motion of the train as realistically as possible in this MMR, the outlines of the trains are reproduced in the corresponding models with a scale ratio of $1 / 8$. The reduced parts in the model include the bogies and wheels on the bottom of the trains. The only exceptions are the model sliders at the two sides of the model; however, the additional area brought on by the sliders in the transverse direction is only about $30 \mathrm{~cm}^{2}$ for each model slider. 
a

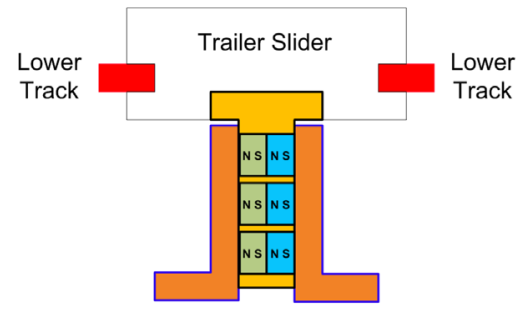

L- Type Steel Plates b

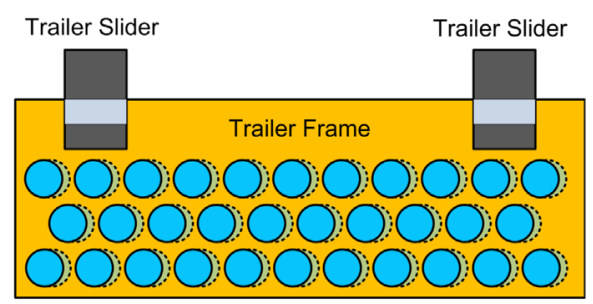

Magnets Eccentrically Mounted on Trailer Frame

Fig. 4. Schematic diagram of the trailer and steel plates for deceleration: (a) cross-sectional view and (b) lateral view.
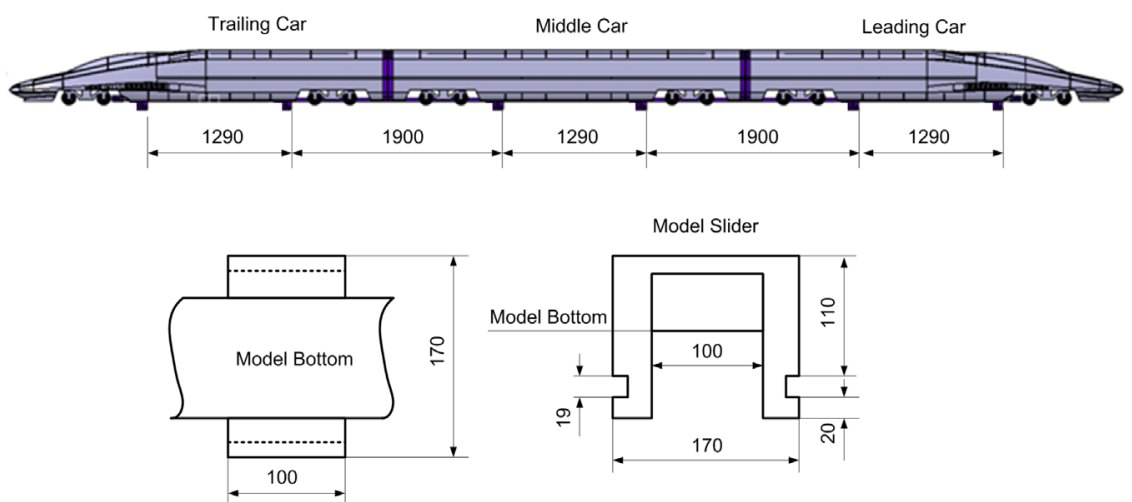

Fig. 5. Schematic diagram of the $225 \mathrm{~kg}$ train model with a leading car, middle car, and trailing car and the positions and the dimension of the model sliders.

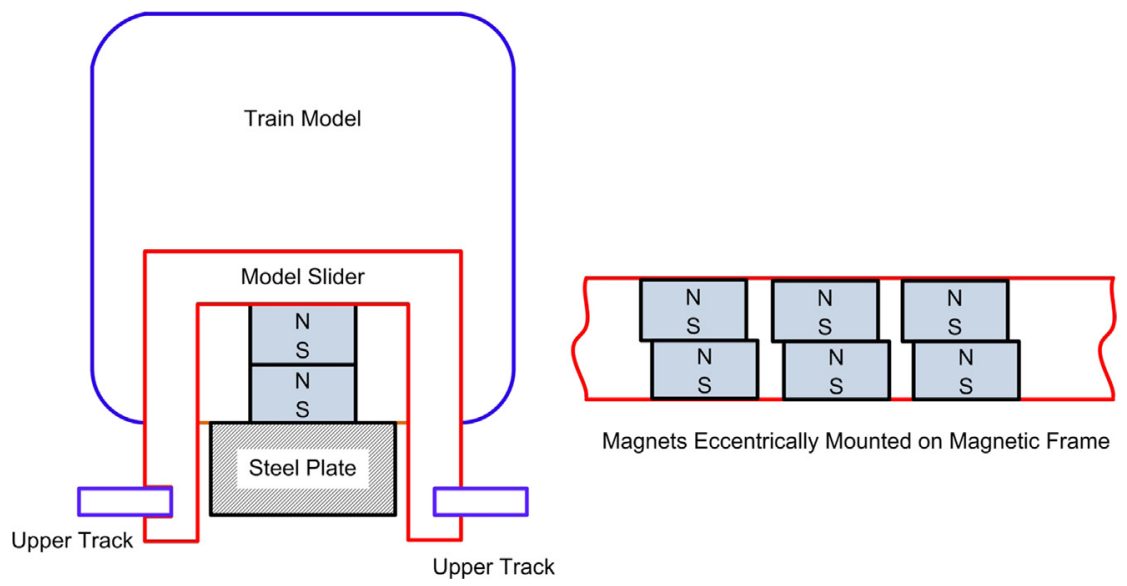

Fig. 6. Schematic diagram of the deceleration of the train model: cross-sectional view and lateral view.

This size is much smaller than the cross section of the train model, which measures about $1600 \mathrm{~cm}^{2}$. The train model used in this work to simulate the passing of a train through a tunnel is shown in Fig. 5, where the train model and slider show identical scale ratios, with the former consisting of a leading car, middle car, and trailing car with a certain outline and a corresponding mass of $225 \mathrm{~kg}$. On their bottoms, six model sliders are used to force the model to slide on the upper track.

As a result of the inertia of the train model, it slides into the testing section, thereby initiating the testing process. During the testing process, the decreasing model speed as a result of the friction between the slide block and tracks and the aerodynamic resistance of the model is neglected because the energy exhausted for the two factors is much lower than the kinetic energy of the model itself. The experimental results and estimation of the aerodynamics reveal that the kinetic energy of the train model is in the order of $10^{5}-0^{6} \mathrm{~J}$. On the other hand, from the actual measurement with a tension meter, the friction between the model slider and the track for the three-car train model with a mass of $265 \mathrm{~kg}$ reaches a level of $10^{2} \mathrm{~N}$ and it exponentially decreases as a common lubricant is applied to the upper track. Moreover, our numerical simulation for the train model with the three-car reveals that the aerodynamic resistance coefficient is about 0.32 and, therefore, the corresponding resistances are in the same order as the friction above. After the model passes through the testing section, it enters the deceleration section with a length of $100.5 \mathrm{~m}$. The brake force of the model comes from the motion of the magnets on the bottom of the model relative to the steel plates.

Although the deceleration of the trailer and train model is based on the obstruction of the motion of the permanent magnets made of NdFeB relative to the steel plates, their forms and structures are different, as shown in Figs. 4 and 6. The poles of the magnets on the bottom of the train model are upward and 


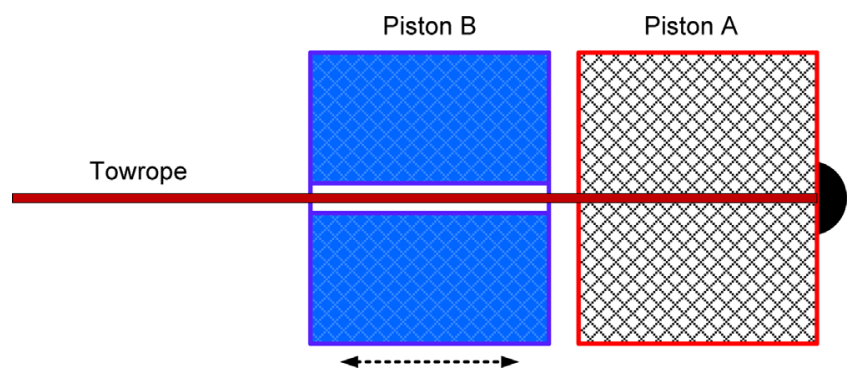

Fig. 7. Schematic diagram of dual pistons in the acceleration tube.

downward, and only one of the magnet poles is used for deceleration. For the trailer, the poles of the magnets are horizontal, and they are all used to produce the brake force. To fix the magnets firmly to the trailer and the bottom of the model, the pairs of holes on both the sides of the magnet frames have the same diameter, but they are not coaxial. After fixing the magnets to the holes using glue, such as acrylic or 502, the two magnets of each pair are tightly attracted to each other; thus, the magnets no longer detach from the frame made of aluminum alloy.

The two pistons are used to prevent the wrapping and knotting of the towrope, which occurs only with one piston as the trailer tends to stop, as presented in Fig. 7. As the trailer comes to an almost full stop, the tension of the towrope still exists for the deceleration of the piston and towrope. The exhaustion of this tension accompanies the violent motion of the piston backward, and this effect results in the serious wrapping and even knotting of the towrope. As another piston, Piston B is stringed on the towrope, the rebound momentum of Piston $A$ and the towrope is transferred to Piston B with the collision of the two pistons. Thus, Piston A tends to be stationary, the towrope remains straight, and Piston B moves backward along the towrope. The total mass of the trailer, towrope, and pistons is $51 \mathrm{~kg}$, and the weight of the magnets in the trailer is $17 \mathrm{~kg}$.

\section{Parameter characteristics and experimental results}

With the train models weighing 106 and $265 \mathrm{~kg}$, the characteristics of the acceleration and deceleration are experimentally investigated for the proposed MMR. The efficiency of the transformation of the air compression energy to the kinetic energy of the trailer and models is also obtained by using the corresponding formula. According to the experimental results on the deceleration distance of the model, the average resistances from the motion of the magnets relative to the steel plates are calculated. To show the reliability and repeatability of the MMR experiments, the evolution of the pressure wave on the tunnel wall at a series of points is measured by pressure sensors as the train model passes through the tunnel model with the same initial pressures. In these experiments, the speeds of the model are measured with a laser beam and a photodiode, which are used along with an oscilloscope to measure the blocking interval as the model keeps out the beam. This device is installed at the beginning of the testing section. The evolution of the acceleration of the model is recorded with an acceleration sensor and a data acquisition device installed in the train model.

Fig. 8(a) shows the dependence of the model speed on the initial compressed air pressure for three cases. In one case, no train model is driven by the trailer in the experiment. The other two cases correspond to the train models weighing 106 and $265 \mathrm{~kg}$. The magnets on the models weigh 36.5 and $54.2 \mathrm{~kg}$. From the numerical analysis of the experimental results, it is concluded that the experimental speed of the three cases is nearly changed with
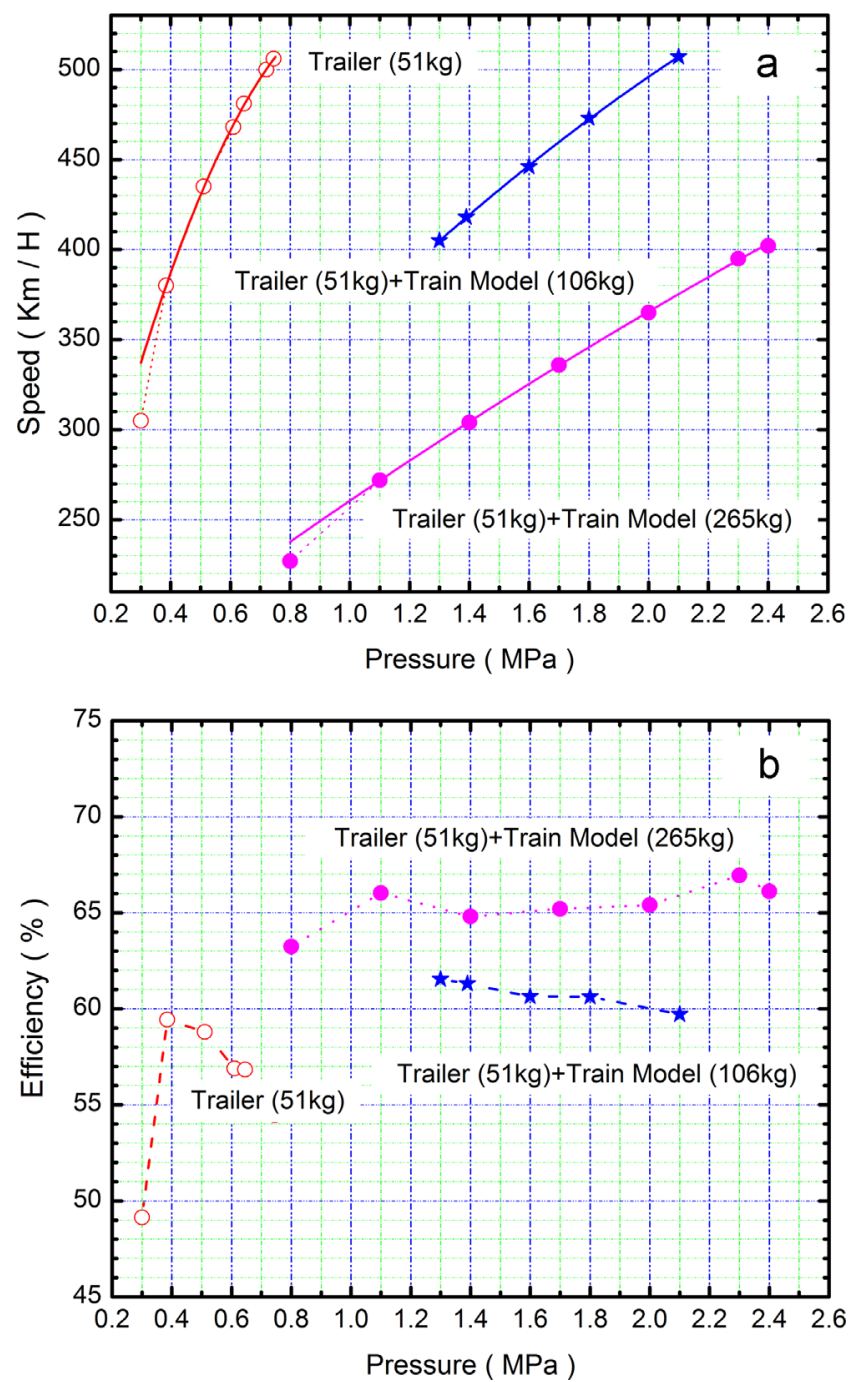

Fig. 8. Experimental results: (a) speed and (b) efficiency of the transformation of air compression energy to kinetic energy versus the initial air pressure.

the initial pressure in quadratic functional form except the lowest speed, respectively, as shown in Fig.8(a).

The expansion of the compressed air in the acceleration process is rapid. Thus, we can use Poisson's equation to estimate the efficiency of the energy transformation from the air compression to the motion of the train model and trailer, in which the heat transfers between the air and the acceleration tube and that between the air and the air chamber are neglected. The efficiency formula for this transformation can be written as

$\eta=\frac{\frac{1}{2}(M+m) v^{2}}{\frac{g P_{0} V_{0}}{\gamma-1}\left[1-\left(\frac{V_{0}}{V_{0}+S L}\right)^{\gamma-1}\right]}$

where $m$ is the sum of the masses of the trailer, towrope, and pistons; and $M$ is the mass of the train model. $v$ is the speed of the model at the end of the acceleration section; it corresponds to the measured results of the speed. $V_{0}$ and $P_{0}$ are the volume of the gas chamber and its initial air pressure, respectively. $S$ and $L$ are the cross-section area of the piston and the acceleration length, respectively, and $\gamma$ and $g$ are the specific heat ratio and the gravity constant, respectively. The parameters of this MMR are as follows: $V_{0}=3.0 \mathrm{~m}^{3}$, $S=0.102^{2} \times 3.14 \mathrm{~m}^{2}, \quad \gamma=1.4, \quad m=51 \mathrm{~kg}, \quad L=54 \mathrm{~m}, \quad M_{1}=0$, $M_{2}=106 \mathrm{~kg}, M_{3}=265 \mathrm{~kg}$, and $g=9.8 \mathrm{~m} / \mathrm{s}^{2}$. The corresponding efficiencies of energy transformation for the three cases versus the initial pressure are shown in Fig. 8(b). The efficiencies range from 50\% 

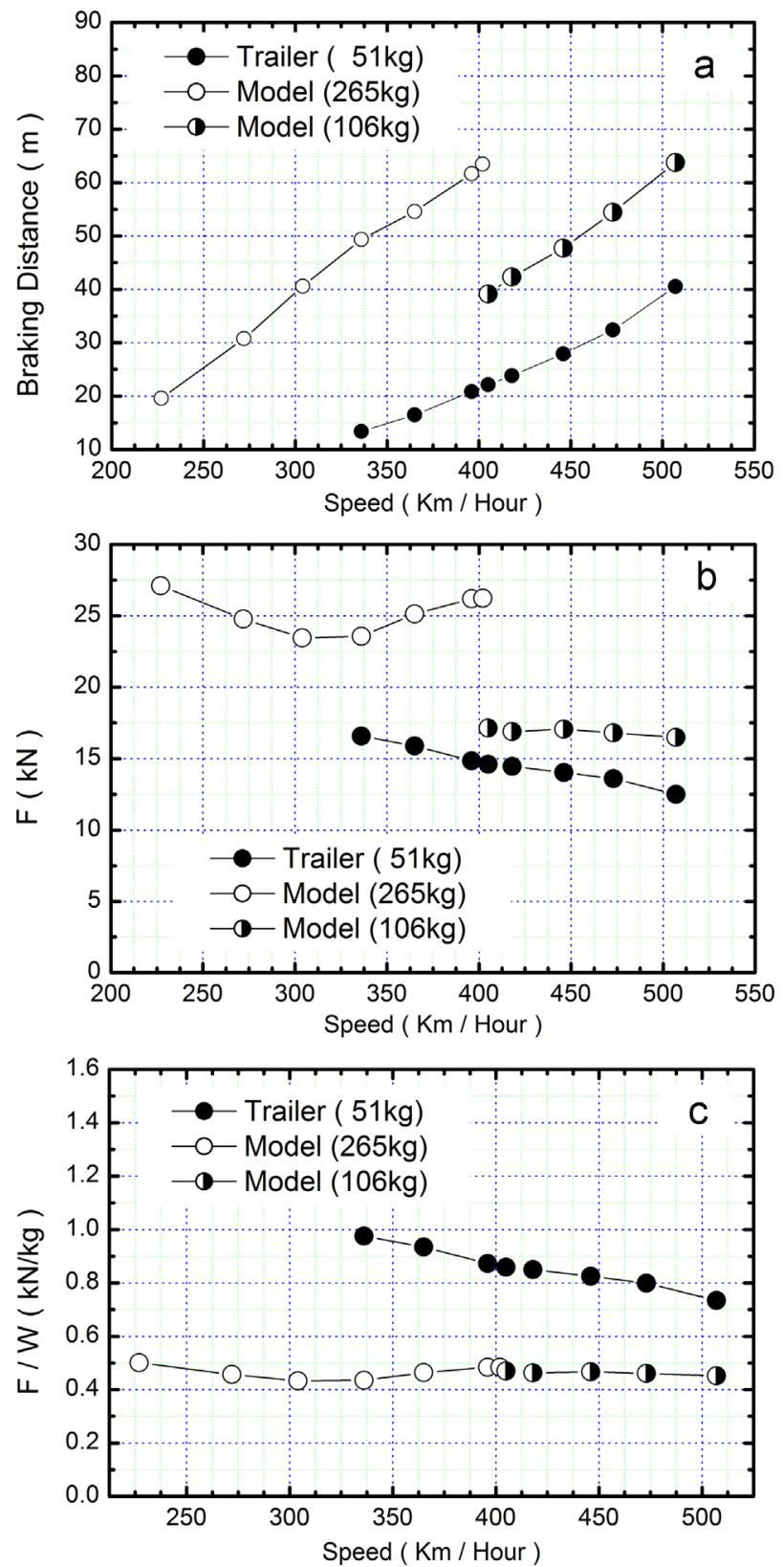

Fig. 9. Deceleration data of the trailer and train model: (a) braking length, (b) total resistance force, and (c) force of the magnets per kilogram.

to $67 \%$. The heavy weight of the model equates to a high efficiency. Without the model, the efficiency ranges from $54.5 \%$ to $59.4 \%$ as the trailer moves at a high speed. The efficiency is higher than $60 \%$ for the model weighing $106 \mathrm{~kg}$. As the weight of the model increases to $265 \mathrm{~kg}$, the average efficiency is approximately $65.4 \%$.

The dependence of the brake distance of the trailer and the models weighing 106 and $265 \mathrm{~kg}$ on speed is shown in Fig. 9(a). The deceleration distance is found to be proportional to the speed, and the errors of the brake distance for one of the models with the same speed fall within $0.6 \mathrm{~m}$, which proves that this form of deceleration for the trailer and train is highly stable. According to the experimental results, the deceleration distance for the train models with masses of 106 and $265 \mathrm{~kg}$ and speeds of 507 and $401 \mathrm{~km} / \mathrm{h}$ is generally shorter than $70 \mathrm{~m}$. On the basis of these data, the acceleration and deceleration capabilities of the train models with different masses can be estimated for this MMR.
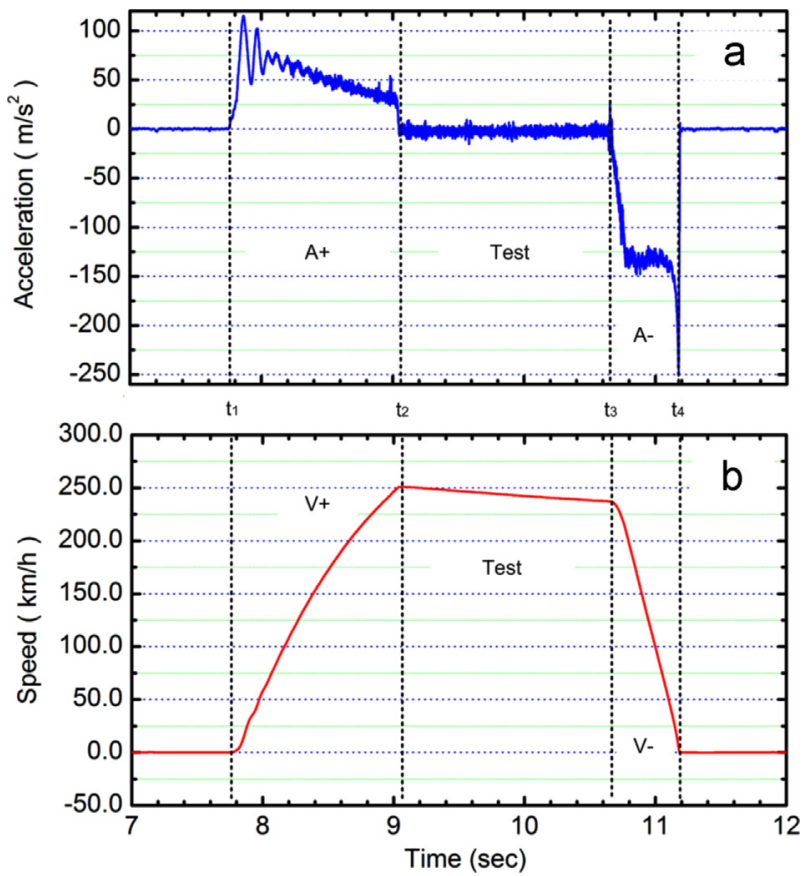

Fig. 10. History of (a) acceleration and (b) speed of the train model (265 kg) with an initial pressure of $0.96 \mathrm{MPa}$.

As the reduction of the kinetic energy of the trailer and train model results from the resistance of the displacement of the magnets to the steel plate. The average values of the forces for the three cases are obtained from the experimental result, as shown in Fig. 9(b). Obviously, magnets with heavy weights equate to strong deceleration forces. On the basis of the data in Fig. 9(a) and (b), the forces of the magnet per kilogram are calculated, and the values of the train model and trailer versus the speed are found to be different, as shown in Fig. 9(c). These forces for the two models are approximately equivalent because of the same structures of the magnet for deceleration shown in Fig. 6. As the two poles of the magnet on the trailer are all utilized to produce the brake force, the values of such brake force per kilogram are twice those of the train models.

Fig. 10(a) shows the typical acceleration history of the train model, in which the initial compressed air pressure is $0.96 \mathrm{MPa}$. The data are recorded by a set of instruments, including an acceleration sensor (ULT27100) and a data acquisition device (LGR5329) installed in the model, where the upper track is not applied with any lubrication. The following three processes of the model can be easily distinguished: acceleration $(A+)$, testing (Test), and deceleration $(\mathrm{A}-)$. An interesting phenomenon is the regular oscillation of acceleration in the acceleration section. Such phenomenon is caused by the towrope between the pistons and the trailer and has been observed in a similar device (Yang et al., 2013). At the beginning of the acceleration, the sudden acceleration of the pistons results in the higher acceleration of the towrope in comparison with the acceleration of the trailer and train model because of the limited elongation of the towrope. The tension wave then propagates forward and backward along the towrope, resulting in this acceleration fluctuation. This phenomenon gradually fades with the continuing entry of the compressed air into the acceleration tube. Finally, the pistons, trailer, and model exhibit identical accelerations. Moreover, the acceleration of the model gradually decreases as the volume of the compressed air increases in the acceleration tube and as the pressure on the pistons decreases. In the brake process, the force of the deceleration suddenly increases and becomes constant, similar to the case in 
a

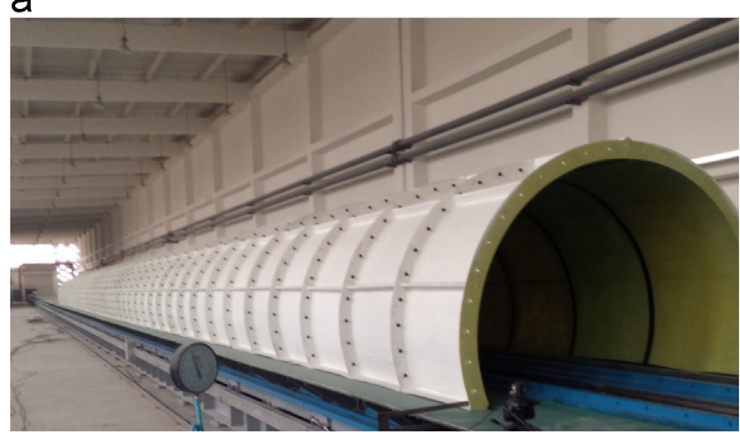

b

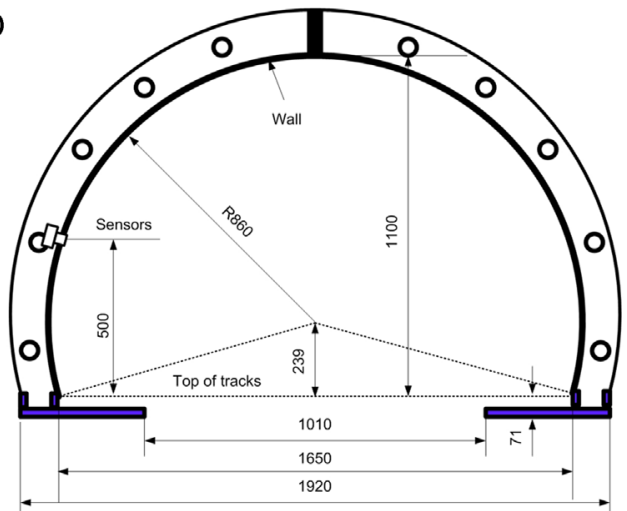

C

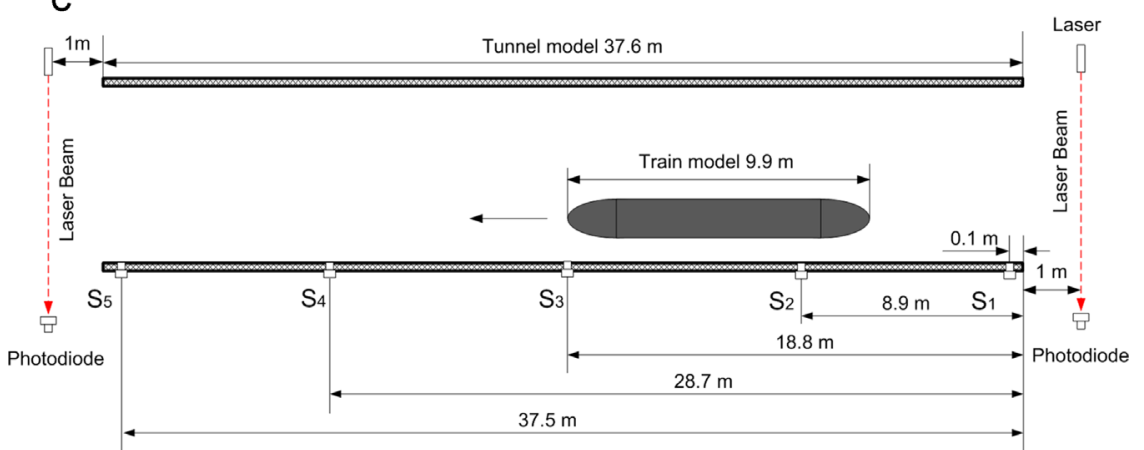

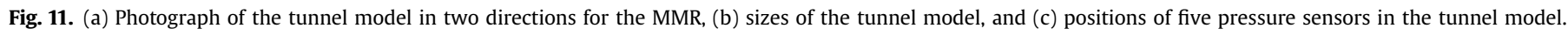

which the train model rapidly enters the deceleration section. As the model moves in this section, the deceleration is constant, which means that the resistance of the magnets does not heavily depend on the speed. Finally, the dramatic rise of the deceleration results from the fact that the stationary friction of the model is larger than the motional friction.

The evolution of the model speed is shown in Fig. 10(b). It is obtained from the time integral process of the acceleration history. First, the increase in the speed is smooth and almost uniform, although the acceleration initially exhibits certain fluctuations. In the testing section, the decrease in the speed should be due to the friction between the slide blocks and the tracks, as well as the aerodynamic resistance. The friction in this case can be reduced by the lubrication on the upper track. Finally, the speed decrease in the deceleration section is uniform because the deceleration of the model from the magnets is approximately constant.

To check the repeatability and reliability of the experimental simulation, the case involving the train model passing through the tunnel model is constructed, as shown in Fig. 11(a). The dimensions of the tunnel model, the positions of the five pressure sensors, and the installation of the tunnel model are displayed in Fig. 11(b) and (c). The speeds of the train model entering and leaving the tunnel are measured by using two sets of measurement instruments for the speed of the train model. Before the experiment, lubrication oil was applied to the upper track. Thus, the decrease in the speed of the train model after passing through the tunnel model is less than $3 \%$. As the speed of the train model as it enters the tunnel model is $232 \mathrm{~km} / \mathrm{h}$, the evolution of the pressure on the tunnel wall is recorded by the five pressure sensors shown in Fig. 11(b) and (c). The train model used in this experiment comprises three cars and weighs $225 \mathrm{~kg}$, as illustrated in Fig. 5. The pressure histories at the positions cited above are shown in Fig. 12. The pressure waves on the tunnel wall in two cases show good agreement. The experiment and corresponding numerical analysis for the passing of the train model through the tunnel and the meeting of two train models in this tunnel model at different speeds are performed successfully. Their results will be introduced and explained in the near future as this paper merely introduces the development of the MMR.

\section{Conclusion}

This paper introduces the principles of the acceleration and deceleration of the train model in a new MMR with a scale ratio of 

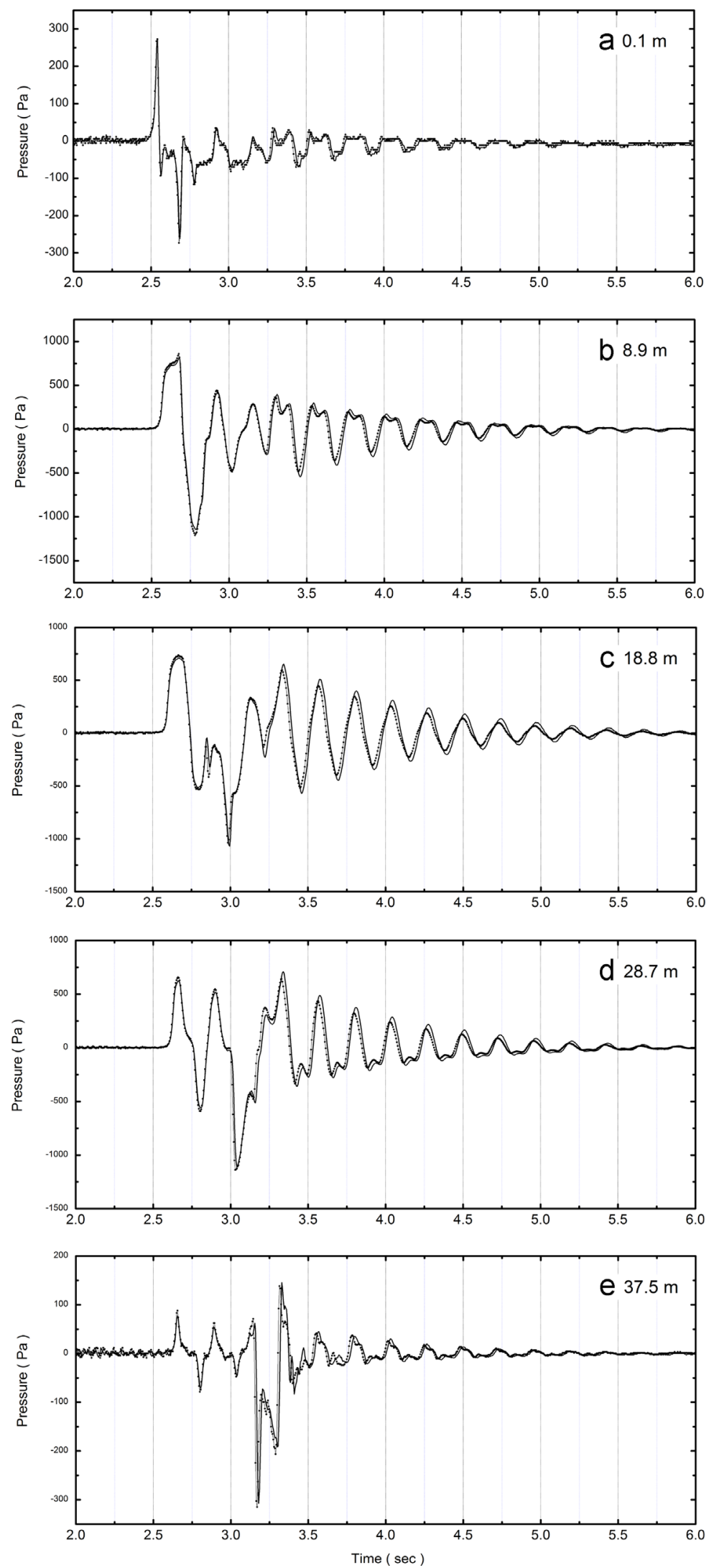

Fig. 12. Evolutions of the pressures at five positions on the tunnel wall as the train model passes through the tunnel model twice at a speed of $232 \mathrm{~km} / \mathrm{h}$.

$1 / 8$. The powers for towing the trailer and train model originate from the pistons in the acceleration tube through a towrope. The pistons are driven by the compressed air from an air gun. On the basis of the resistance between the magnets and the steel plate during motion, the magnetic braking devices for the trailer and model are proposed and developed. The experimental results 
reveal that the train models weighing 265 and $106 \mathrm{~kg}$ can be accelerated to speeds of 401 and $507 \mathrm{~km} / \mathrm{h}$, respectively, and then safely decelerated. Under such large driving capacity, a train consisting of one or several cars can be reproduced at a scale ratio of $1 / 8$ for experimental simulations. To demonstrate the repeatability of the experiment on the MMR under the same initial condition, the measured results for the pressure history are introduced through the case in which the train model passes through the tunnel model.

\section{Acknowledgements}

The work was supported by the National Key Technology R\&D Program (Grant No. 2009BAG12A03) and the National Natural Science Foundation of China (Grant No.10472123).

\section{Appendix A. Supplementary material}

Supplementary data associated with this article can be found in the online version at http://dx.doi.org/10.1016/j.jweia.2016.03.002.

\section{References}

Demmenie E., De Bruin A., Klaver E., 1998. Experimental pressure wave research at NLR for high-speed rail tunnels, National Aerospace Laboratory Nlr, Technical Publication TP98375.

Doi, T., Ogawa, T., Masubuchi, T., Kaku, J., 2010. Development of an experimental facility for measuring pressure waves generated by high-speed trains. J. Wind Eng. Ind. Aerodyn. 98 55-1.
Endo, H., Meguro, F., Ota, M., Maeno, K., 2014. Small model experiment on the gradient of pressure wave by entering the tunnel of a conventional limited express. J. JSEM 14, s42-s47.

Jirout, M., Mack, W., Lugner, P., 2009. Non-smooth dynamics of a magnetic track brake. Regul. Chaotic Dyn. 14, 673-681.

Johnson, T., Dalley, S., 2002. TRANSAERO - A European Initiative on Transient Aerodynamics for Railway System Optimisation. Springer-Verlag, Berlin Heideberg, pp. 123-135.

Jou, M., Shiau, J., Sun, C., 2006. Design of a magnetic braking system. J. Magn. Magn. Mater. 304, e234-e236.

Ma, D., Shiau, J., 2010. The design of eddy-current magnet brakes. Trans. Can. Soc. Mech. Eng. 3, 19-37.

Maclatchy, C., Backman, P., Bogan, L., 1993. A quantitative magnetic braking experiment. Am. J. Phys. 60, 1096-1101.

Ozawa S., Maeda T., 1988. Model experiment on reduction of micro-pressure wave radiated from exit. In: Proceedings of the International Symposium on Scale Modeling, The Japan Society of Mechanical Engineers, Tokyo, July, pp. 18-22.

Peters, J.L., 1983. Aerodynamics of high speed trains and Maglev vehicles. In: Dorgham, M.A. (Ed.), Impact of Aerodynamics on Vehicle Design. Inderscience, London, pp. 308-341.

Raghunathan, R.S., Kim, H.D., Setoguchi, T., 2002. Aerodynamics of high-speed railway train. Prog. Aerosp. Sci. 38, 469-514.

Sasoh, A., Matsuoka, K., Nakashio, K., et al., 1998. Attenuation of weak shock waves along pseudo perforated walls. Shock Waves 8, 149-159.

Schetz, J.A., 2001. Aerodynamics of high speed trains. Annu. Rev. Fluid Mech. 33 $371-414$.

Takayama, K. Sasoh, A., Onodera, O., Kaneko, R., Matsui, Y., 1995. Experimental investigation on tunnel sonic boom. Shock Waves 5, 127-138.

Tian, H.Q., 2007. Train Aerodynamics, 99-114. China Railway Publish House, Beijing, pp. 268-286 (In Chinese).

Wagner, C., Horstmann, G., Herzog, S., Jakubek, D., Rutschmann, S., 2014. Shape optimization of train heads with respect to the aerodynamic loads on track side objects. Int. J. Railw. Technol. 3, 83-104.

Yang, Q.S., Song, J.H., Li, D., Zhang, J., Yang, G.W., 2013. Train model acceleration and deceleration. Sci. China Tech. Sci. 56, 642-647.

Zhou, D., Tian, H.Q., Zhang, J., Yang, M.Z., 2014. Pressure transients induced by a high speed train passing through a station. J. Wind Eng. Ind. Aerodyn. 135, 1-9. 